\title{
Functional outcomes associated with expiratory muscle strength training: Narrative review
}

\author{
Helena Laciuga, MA; ${ }^{1 *}$ John C. Rosenbek, PhD; ${ }^{1}$ Paul W. Davenport, PhD; ${ }^{2}$ Christine M. Sapienza, PhD $^{\mathbf{3}}$ \\ Departments of ${ }^{1}$ Speech, Language, and Hearing Sciences, and ${ }^{2}$ Physiological Sciences, University of Florida, \\ Gainesville, FL; ${ }^{3}$ College of Health Sciences, Jacksonville University, Jacksonville, FL; Brain Rehabilitation Research \\ Center, Malcom Randall Department of Veterans Affairs Medical Center, Gainesville, FL; and Brooks Rehabilitation \\ Hospital, Jacksonville, FL
}

\begin{abstract}
This review presents the available evidence for the effects of expiratory muscle strength training (EMST) with the use of a pressure threshold device. The investigators used computerized database searches for studies reporting the outcomes of pressure threshold EMST published after 1994. A total of 24 selected articles presented outcomes related but not limited to respiratory function, such as speech, swallow, voice, and cough function in persons with neurologic conditions such as Parkinson disease, multiple sclerosis, and Lance-Adams syndrome; in persons with respiratory diseases, such as chronic obstructive pulmonary disease; and in healthy young adults and sedentary and active elderly. Several studies demonstrated promising outcomes of EMST as a non-task-specific training for airway protection in persons with dysphagia secondary to neuromuscular impairments; however, further research is needed to confirm and generalize the reported findings.
\end{abstract}

Key words: COPD, cough air flow, dysphagia, dysphonia, dyspnea, exercise, expiratory, muscle strength training, maximum expiratory pressure, neuromuscular diseases, neuroplasticity, quality of life, respiration.

\section{INTRODUCTION}

Expiratory muscle weakness results from various peripheral and central conditions affecting the neuromuscular system (e.g., [1-6]). In particular, reduced expiratory muscle strength may decrease the ability to generate adequate expiratory pressure for voice production or airway clearance during cough. Expiratory muscle strength training (EMST) is an exercise program designed to

Abbreviations: $6 \mathrm{MWT}=6 \mathrm{~min}$ walk test, ALSS $=$ Amyotrophic Lateral Sclerosis Severity Scale, BDI = baseline dyspnea index, $\mathrm{BP}=$ blood pressure, $\mathrm{CES}=$ Communicative Effectiveness Survey, COPD = chronic obstructive pulmonary disease, $\mathrm{EMST}=$ expiratory muscle strength training, $\mathrm{FEV1}=$ forced expiratory volume within $1 \mathrm{~s}, \mathrm{FVC}=$ forced vital capacity, HR = heart rate, IMST = inspiratory muscle strength training, LAS $=$ Lance-Adams syndrome, MPT $=$ maximum phonation time, $\mathrm{MRC}=$ Medical Research Council, $\mathrm{MS}=$ multiple sclerosis, $\mathrm{P}-\mathrm{A}=$ penetration-aspiration, $\mathrm{PCF}=$ peak cough flow, $\mathrm{PD}=$ Parkinson disease, PEFR $=$ peak expiratory flow rate, $\mathrm{PEmax}=$ maximum expiratory pressure, PEmpeak $=$ peak expiratory pressure, $\mathrm{PI}=$ pulmonary index, $\mathrm{PImax}=$ maximum inspiratory pressure, PPIA $=$ postpeak plateau integral amplitude, $\mathrm{sEMG}=$ surface electromyography, $\mathrm{SpO}_{2}=$ oxygen saturation, $\mathrm{SVP}=$ sustained vowel prolongation, $\mathrm{SWAL}-\mathrm{QOL}=$ dysphagia-specific quality of life, UES = upper esophageal sphincter, UPDRS III = Unified Parkinson Disease Rating Scale, $\mathrm{VA}=$ Department of Veterans Affairs, $\mathrm{VE}=$ minute ventilation, $\mathrm{VRQOL}=$ voice-related quality of life, $\mathrm{WPM}=$ words per minute.

"Address all correspondence to Helena Laciuga, MA; 336 Dauer Hall, University of Florida, Gainesville, FL 32611; 352-392-2113; fax: 352-846-0243; Email: hlaciuga@ufl.edu http://dx.doi.org/10.1682/JRRD.2013.03.0076 
modify physiological mechanisms associated with expiratory tasks. A principal therapeutic goal of EMST is strengthening the muscles of the expiratory pump by increasing expiratory load during breathing exercises using either resistive or pressure threshold devices.

Although multiple studies have investigated the outcomes of EMST, no classification of evidence concerning this treatment type has been found in the published literature within the last $19 \mathrm{yr}$. Presenting the amount and quality of evidence for this type of training may help clinicians make decisions about its appropriate application.

This article provides a comprehensive review of available literature presenting research evidence for effects of EMST. The purpose of this assessment is not a systematic review, but rather a categorization of available evidence on this specific training modality applied in multiple conditions or diseases. We selected studies that used the pressure threshold expiratory muscle training devices with a consistent numeric measure because this allowed comparison of the outcomes across multiple sources of evidence. For the purposes of this review, a pressure threshold device was considered more reliable than a resistive expiratory muscle training device. The pressure threshold device allows the amount of expiratory load to be defined and controlled, which is helpful in establishing therapeutic goals and assessment of patients' progress [7-8].

\section{METHODS: STUDY SELECTION CRITERIA}

The literature for this review consisted of studies published after 1994. The PubMed, Cochrane, and Google Scholar databases were searched using the phrases "expiratory muscle training" and "expiratory muscle strength training." All the articles uncovered after the initial search were further reviewed to determine the use of an expiratory pressure threshold device. A total of 24 articles were selected based on the predetermined criteria. Among the reviewed studies, the majority were published in American scientific journals, with the exception of two international publications, one from the Journal of Physical Therapy Science [9] and one from Respiratory Medicine [10].

\section{RESULTS}

\section{EMST Device and Training Protocols}

The mechanism that creates the expiratory load in a pressure threshold device is a spring-loaded relief valve housed inside the device. The pressure threshold relief valve opens when a sufficient expiratory pressure is generated by the user during a forceful expiration into the device's mouthpiece [11]. The prototype of the EMST device was an inspiratory muscle trainer with a modified spring to produce higher pressure loads ranging from 15 to $125 \mathrm{cmH}_{2} \mathrm{O}$, with use of the opposite side of the device for expiration [12]. The threshold pressure used for EMST ranges from 30 percent [12] to 75 percent [11] of the trainee's maximum expiratory pressure (PEmax) at total lung capacity.

The EMST protocol is not standardized and varies by intensity and duration across the reviewed studies. Suzuki et al. reported the effects of EMST based on expirations through the threshold device twice daily at the user's own breathing frequency and tidal volume for 15 min for $4 \mathrm{wk}$ [12]. Weiner at al. trained participants with

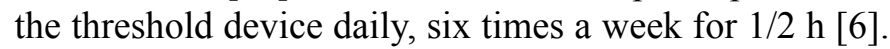
During the first week, the expiratory load was set to 15 percent of the user's PEmax. The following week, the load was increased by 5 to 10 percent each session up to 60 percent of the user's baseline PEmax at the end of the first month. The load was maintained at the level of 60 percent of the user's PEmax throughout the training period. In a study by Mota et al. [10], participants completed a 5 wk EMST program including 30 min sessions, three times a week. Participants exhaled through the threshold device at 50 percent of their PEmax following cycles of $3 \mathrm{~min}$ of EMST breathing and 2 min rest. Sapienza et al. proposed five cycles of five expirations through the EMST device $5 \mathrm{~d} / \mathrm{wk}$ for $4 \mathrm{wk}$ [11], which was also applied by Pitts et al. [4] and Troche et al. [13]. Baker et al. modified the EMST protocol and studied the effects of the EMST after 4 and 8 wk training periods [14]. Saleem et al. applied EMST in a single case study for 20 wk [15], and finally, Anand et al. studied training frequencies of 1,3 , and $5 \mathrm{~d} /$ wk using the EMST device [16].

\section{Effects of EMST on Expiratory Muscle Strength and Respiratory Function}

\section{Chronic Obstructive Pulmonary Disease}

Three studies examined the outcomes of the EMST on persons with chronic obstructive pulmonary disease 
(COPD). Weiner et al. studied the effects of EMST on expiratory muscle strength and endurance, exercise performance, and the sensation of dyspnea in patients with severe COPD and significant chronic airflow limitation by comparing a treatment group against a sham group [6]. Participants of both groups exhaled through the expiratory port of the Threshold Inspiratory Muscle Trainer (Healthscan Products Inc; Cedar Grove, New Jersey). The treatment group used a device with the expiratory load modified from 15 to 60 percent of their PEmax, and the sham group was trained with a low load of $7 \mathrm{cmH}_{2} \mathrm{O}$ [6].

Weiner et al. reported statistically significant changes in the treatment group: an increase $(21 \%)$ in the PEmax, an improvement in the expiratory muscle endurance based on the peak expiratory pressure (PEmpeak) increase, and a small but statistically significant increase $(19 \%)$ in the distance walked during the 6 min walk test (6MWT) [6]. The results also demonstrated a small but insignificant decrease in dyspnea. Although Weiner et al. emphasized the association between decreased expiratory muscle performance and both reduced exercise tolerance and reduced quality of life in patients with COPD, participants' quality of life was not assessed in the study [6]. The trial included only normocapnic patients and did not compare EMST with other forms of training, which the authors considered the study limitation [6].

In another study involving participants with severe COPD, Weiner et al. evaluated the impact of inspiratory muscle strength training (IMST) and EMST on respiratory muscle strength and endurance, exercise performance, and sensation of dyspnea [17]. For this experiment, participants with COPD and significant chronic air flow limitation were randomized into four groups: EMST, IMST, a combination of EMST and IMST, or EMST with a low load of $7 \mathrm{cmH}_{2} \mathrm{O}$ (control group) using the Threshold Inspiratory Muscle Trainer.

The authors reported significant increases in PEmax and PEmpeak within the EMST and EMST + IMST groups. Improvements in inspiratory muscle strength were associated with improvements in exercise performance and decreased sensation of dyspnea in daily activities, while an increase in expiratory muscle strength was correlated with a significant increase in exercise performance alone. The increase in the Mahler Baseline Dyspnea Index (BDI), a measure of dyspnea in daily activities, was noticed in the IMST and EMST + IMST groups, with no change in BDI in the EMST and control groups. The study demonstrated a significant improve- ment in 6MWT in all three treatment groups, but not in the control group. Interestingly, a combination of IMST and EMST did not contribute to improved exercise performance and the reduction of sensation of dyspnea when compared with IMST alone. On the basis of the study results, the investigators concluded that IMST might be the only respiratory muscle training to improve both dyspnea and exercise performance in patients with COPD [17].

The effects of 5 wk of EMST on lung function, exercise tolerance, dyspnea, and health-related quality of life in males with severe COPD were evaluated by Mota et al. [10]. The investigators performed lung function assessment, which included forced spirometry, inspiratory capacity, maximal voluntary ventilation, plethysmographic lung volumes, carbon monoxide transfer coefficient, arterial blood gases, and PEmax. For exercise tolerance, 6MWT, leg cycloergometry, arm cycloergometry, degree of dyspnea on a Borg scale, and distance walk were applied. Dyspnea during daily activities was evaluated with the modified Medical Research Council (MRC) scale, and quality of life was assessed with the St. George's Respiratory Questionnaire. The training group used a threshold device with a load corresponding to about 50 percent of the participants' PEmax, while the sham group was trained with no additional expiratory load. The initial load for the sham group was not specified. Mota et al. reported a significant post-EMST increase (19\%) in expiratory muscle strength correlated with the improvement in exercise performance and a reduction of dyspnea at rest and with exertion [10]. A significant increase in PEmax (13\%) was observed in the $6 \mathrm{MWT}$ in the training group. In addition, a noticeably lower level of dyspnea during the 6MWT was reported in the training group compared with the sham group. A training group improved maximal workloads in either leg or arm cycloergometries and demonstrated significantly greater decrease in exertional dyspnea MRC scale score than the sham group. A correlation of a post-EMST exertional dyspnea measured at the end point of the 6MWT and the improvement in health-related quality of life ( $r=0.634$, $p<0.05)$ were considered the most clinically relevant findings [10]. According to the authors' speculations, the EMST could have improved cough effectiveness for more efficient clearance of the airways; however, this component was not assessed in the study. When designing the study, the investigators did not expect major changes in cough because the participants were clinically 
stable and nonbronchorrheic, with a daily sputum volume less than $30 \mathrm{~mL}$.

\section{Multiple Sclerosis}

The effects of the EMST on expiratory muscle strength and endurance were reported for patients with multiple sclerosis (MS) by Smeltzer et al. [8]. They examined changes of PEmax and maximum inspiratory pressure (PImax) after 1, 2, and 3 mo of an intervention with the pressure Threshold Inspiratory Muscle Trainer. All the patients demonstrated decreased expiratory muscle strength at baseline, with PEmax ranging between 45 and 60 percent predicted. Participants were randomly assigned to an EMST training group or a sham training group. The initial training threshold load was determined by decreasing the required expiratory pressure by 10 percent of a participant's PEmax and lowered by 10 percent after each unsuccessful attempt to open the pressure valve. Participants completed three sets of 15 breaths into the pressure threshold device twice a day for $3 \mathrm{mo}$, with the pressure threshold gradually increased based on participants' progress. The sham group received training using the same device, but with a small inspiratory load incremented periodically within the 3 mo trial. There was a significant increase of PEmax in the EMST group after 3 mo of training, which indicated at least partial conditioning of expiratory muscles in patients with MS. This result was promising for EMST use in patient groups for improvement of cough effectiveness and airway clearance, decreased breathing work, and increased respiratory reserve [8].

\section{Parkinson Disease}

Among the evaluated studies examining EMST effects on expiratory muscle strength in patients with Parkinson disease (PD), the earliest evidence was reported in a single case study by Saleem et al. [15], who evaluated the effects of a $20 \mathrm{wk}$ EMST program in a 54 yr-old woman with tremor-predominant early idiopathic PD. The investigators assessed posttraining changes in PEmax and changes in Unified Parkinson Disease Rating Scale (UPDRS III) score. Saleem et al. hypothesized that a pattern of adaptation in expiratory muscles would be comparable to the adaptations in limb muscles [15]. The study results showed a 158 percent increase in PEmax from baseline at the 20th week of the training. After 4 wk of detraining, the participant demonstrated only 16 percent reduction in PEmax; however, her
PEmax remained 104 percent above baseline. The motor examination score of the UPDRS III, which assesses the symptoms of PD, improved constantly during the $20 \mathrm{wk}$ training period and worsened during the detraining period to the level presented at the fourth week of EMST. The patterns of observed responses to the $20 \mathrm{wk}$ EMST program suggested that the slow adaptation pattern was specific to the expiratory muscles and not induced or affected by the process of PD [15].

Another assessment of the effects of EMST on expiratory muscle strength in PD was conducted by Silverman et al. [18]. This preliminary study evaluated a degree of expiratory muscle weakness in one group of 28 participants with PD and examined the effects of EMST on inspiratory and expiratory muscle strength in three participants with PD. The investigators identified impairments of respiratory muscle function in the studied participants by comparing the obtained values of PEmax and PImax to normative data from the study of Enright et al. [19]. A training protocol and the expiratory load used in the training were not included in the report. The study reported substantial increases in PEmax for all three participants by $91.3,75.3$, and 79.5 percent. Similarly, significant increases in PImax by 82.9, 120, and 54.4 percent were also observed [18], although inspiratory muscle strength was not the primary target in the training. The significant increase in respiratory muscle strength observed during the relatively short, $4 \mathrm{wk}$ training period was considered similar to the response of the limb muscles to exercise and was presumed by the investigators to be related to neural adaptations including improved organization of motor units, improved inter- and intramuscle coordination, increased central nervous system activation, and more efficient neural receptor patterns [18].

\section{Healthy Adults}

A number of studies included in this review assessed physiological mechanisms associated with EMST in healthy adults with a wide age range. Suzuki et al. [12] conducted an experimental study based on a hypothesis that increasing expiratory muscle strength would reduce the magnitude of the central command needed from the expiratory muscles during exercise when ventilation was increased. The investigators expected that an improvement in expiratory muscle strength following $4 \mathrm{wk}$ of EMST would reduce the sensation of respiratory effort during treadmill exercises. They examined healthy adults randomly assigned to a 4 wk EMST group or a control 
group. The training group used the Threshold Inspiratory Muscle Trainer converted to an expiratory device with an expiratory load of 15 to $125 \mathrm{cmH}_{2} \mathrm{O}$ and with the expiratory threshold set at 30 percent of the individual's PEmax. The EMST application resulted in increased expiratory muscle strength and reduced sensation of respiratory effort during exercise with no change in the relationship between the sensation of respiratory effort and minute ventilation (VE). Specifically, the increase of PEmax at function residual capacity was 20.2 percent after 2 wk of EMST and 18.2 percent after 4 wk of EMST. Suzuki et al. reported less increase in respiratory effort after EMST as the degree of exercise increased, a decrease in VE and breathing frequency during exercise, and an increase in expiratory time after EMST [12]. The findings suggest that EMST diminishes the sensation of respiratory effort during exercise by reducing VE [12]. The expiratory load used for this study was 30 percent of a participant's PEmax, which could be considered insufficient to induce maximal improvements in expiratory muscle strength and to observe greater effect of expiratory muscle strengthening on the sensation of respiratory effort.

The effects of EMST on pulmonary function and peak cough flow (PCF) in healthy adults were examined by Sasaki et al. [20]. Thirty-three healthy adults were randomized into three groups: group 1, which received the EMST at a natural respiratory flow rate; group 2, which received the EMST at a fast respiratory flow rate; and group 3, which served as a control group. The investigators used the Threshold PEP (Healthscan Products Inc) at 30 percent of participant's PEmax for training purposes. They measured pre- and posttraining values of participants' PEmax, forced vital capacity (FVC), forced expiratory volume within $1 \mathrm{~s}$ (FEV1), peak flow rate, and PCF. Both training groups demonstrated significant postEMST improvements in PEmax regardless of the expiratory flow rate used in the training. The end point values of PEmax did not differ significantly in the two groups trained at 30 percent PEmax, suggesting that the flow rate was not a critical factor in the training protocol at this level of the expiratory load. There was no post-EMST change in lung function parameters, including PCF. These findings may not necessarily apply to patient populations, especially the lack of changes in PCF. Since the mean baseline value of PCF was relatively high $(8-9 \mathrm{~L} / \mathrm{s})$ in this participant group, the capacity for the improvement could have been limited [20].
Sapienza et al. compared changes in PEmax between healthy high school wind instrument players who received the EMST and wind instrument players who formed a control group [11]. Significant post-EMST improvements in expiratory muscle strength were reported for both sexes in the trained group, and interestingly, the control group demonstrated a decrease in PEmax. The investigators suggested that a relatively short, 2 wk exercise regimen can result in neural adaptations to training, with increased neuromuscular excitability, enhanced coordination, improved ability to obtain maximum vital capacity, and more efficient motor programming [11].

The healthy young adults participating in Baker et al.'s study were assigned to either a 4 wk training (group 1) or $8 \mathrm{wk}$ training (group 2) using the EMST program [14]. The study purpose was to assess respiratory outcomes expressed in PEmax, FVC, and FEV1 following EMST training and a detraining period. Following the training period, an increase in PEmax by 41 percent was observed in group 1 and by 51 percent in group 2. In addition, the authors reported decreases in PEmax by 7 percent in group 1 and by 10 percent in group 2, as measured from the end of the training to the end of the detraining period. No significant changes were observed in pulmonary function measures after the training period. Based on the results of the study, the investigators concluded that shorter training durations can produce equally significant increases in muscle strength with minimal deteriorations within a 2 mo detraining period [14].

Griffiths and McConnell [21] compared the effects of EMST, IMST, and a combination of both training types on respiratory function and rowing performance in competitive male rowers ranked according to their ergometer rowing performance over 6 min and divided into EMST and IMST training groups. The participants completed 4 wk of IMST or EMST followed by 6 wk of combined IMST and EMST. Pre- and posttraining measurements included PImax, PEmax, VE, maximal flow volume loops, peak inspiratory flow, peak expiratory flow rate (PEFR), FVC, FEV1, and forced expiratory flow at 50 percent of FVC, pulmonary gas exchange, and a progressive incremental rowing exercise test [6]. The mean PImax in the IMST group was 26 percent higher after 4 wk of IMST and improved further after the combined IMST/ EMST phase to 30 percent relative to baseline. There was also significantly higher PImax (13\%) after the combined IMST and EMST in the EMST group. The study demonstrated an improvement in PEmax to 31 percent in the 
EMST group during the combined IMST and EMST as compared with PEmax at baseline. In general, the study showed that IMST improved rowing performance more than EMST did regardless of the post-EMST improvements in PEmax. Interestingly, no significant changes were observed in breathing pattern or the metabolic response during the exercise test in either group [21]. The investigators explained that changes to expiratory muscle function failed to induce changes in performance because the potency of the expiratory muscle metaboreflex is lower than the potency of the inspiratory muscle metaboreflex. They also speculated that the threshold of the expiratory muscle metaboreflex is higher than the threshold of the inspiratory muscles and is not activated by maximal rowing [21].

One of the selected experimental studies examining effects of EMST involved modifications of training intensity and duration to assess the changes in expiratory muscle strength in healthy participants. Anand et al. recruited healthy, young participants and randomized them into two groups to receive $5 \mathrm{~d} /$ wk training or $3 \mathrm{~d} / \mathrm{wk}$ training [16]. The results showed significant posttraining increases in PEmax in both treatment groups with no significant mean difference in PEmax between the groups. According to Anand et al. [16], the significant improvement in PEmax in both groups with nonsignificant postEMST differences in PEmax between groups indicates that decreasing EMST frequency will not diminish the training benefits, but may become less time consuming with equally significant outcomes. This study raises the question for future research of whether modifying the number of repetitions within each session and changes in training intensity may eventually establish the optimal dosage of expiratory muscle exercises.

\section{Effects of EMST on Cough and Swallow Function}

\section{Cystic Fibrosis, Chronic Obstructive Pulmonary \\ Disease, Multiple Sclerosis, and Healthy Individuals}

Both cystic fibrosis and COPD result in excessive mucus build-up in the lower airways. For persons with these diseases, strong, effective cough is particularly important for the ability to clear the airways from secretions. One of the serious complications of MS, on the other hand, is dysphagia often leading to aspiration pneumonia. However, the risk of developing aspiration pneumonia may be reduced with the increase of cough effectiveness.
Our classification included one meta-analysis of the effects of coughing exercises and EMST in enhancing cough function by Kojima et al. [9]. Coughing exercises in the evaluated studies were completed by either coughing alone, a forced expiration technique, or an assisted coughing method. The analysis was performed on 10 selected randomized trials: 6 investigating coughing exercise in patients with cystic fibrosis and COPD and 4 focused on EMST with healthy individuals, patients with MS, and persons with COPD. The outcome variables used to report the effects of cough exercises were weight of sputum expectorated and radioactive aerosol clearance, while PEmax and PImax were the measures of preand post-EMST.

According to Kojima et al. [9], the reviewed studies reported significant increases in PEmax following EMST. From this meta-analysis, however, it could not be directly determined whether EMST enhanced cough effectiveness because none of the selected studies used PCF to assess cough strength. The only indicator of cough enhancement was the increase of sputum weight reported after cough exercises. Most of the selected studies had small sample sizes; demonstrated heterogeneity of groups in terms of age, sex, body mass index, FEV1, PEFR; and included various diseases with different origins of the airway dysfunction.

\section{Multiple Sclerosis}

In addition to the respiratory function, cough efficacy has been assessed after intervention with EMST in persons with MS. Gosselink et al. investigated the effects of EMST on respiratory muscle function, FVC, and cough efficacy in patients randomly assigned to a training group or a control group [1]. Pre- and post-EMST measurements included PEmax, FVC, and pulmonary index (PI) following 3 mo of EMST with the Threshold Inspiratory Muscle Trainer. For training purposes, the expiratory load was set to 60 percent of participants' PEmax.

Gosselink et al. reported significant post-EMST improvements in a treatment group in PImax compared with baseline and substantial but not statistically significant improvement in PEmax [1]. The treatment group demonstrated significant improvement in the objectively and subjectively rated cough efficacy based on the participants' PI scores compared with baseline and with the control group. Gosselink et al. used a perceptual rating scale to assess cough efficacy with no instrumental cough flow measurement [1]. The Index of Pulmonary 
Dysfunction in MS is a questionnaire consisting of two sections: the first section requires the patient's self-rating of the ability to handle secretions and reduction in cough strength; the second section uses the examiner's rating of the patient's cough strength and the number of words the patient can produce when counting aloud on a single exhalation. The examiner's rating is predominantly based on perceptual evaluation of cough loudness and an assessment of expiratory function by counting the number of words produced without using any specific time unit [1]. The investigators admitted that a limitation of the methods was the participants' weakness and frequent inability to follow instructions. The values of FVC and mouth pressure at specific lung volumes were either imprecise or missing. For example, values below $7 \mathrm{cmH}_{2} \mathrm{O}$ were not accepted; therefore, the authors expressed concern that the inspiratory or expiratory muscle weakness could have been underestimated.

Chiara et al. investigated the training and detraining effects of EMST on respiratory function and voluntary cough in participants with MS with mild to moderate disability and age- and sex-matched healthy individuals [22]. The analyses were focused on pre- to posttraining changes in values of PEmax, FVC, FEV1, and PEFR of maximal voluntary cough and on the variability of PEmax in relation to the independent variables. The only significant post-EMST results observed in this study were the increases in PEmax and PEFR, which remained significantly above baseline following a posttraining period in both groups. Although the EMST did significantly improve the maximal cough parameters in the patients with MS and healthy individuals, patients with moderate disability demonstrated significantly greater pretraining to posttraining and pretraining to detraining increases of PCF than patients with mild disability. The changes in PEmax explained only changes in the expiratory rise time in cough at a significant level. Despite 90 percent participant compliance, the level of expiratory effort was not observed at each session; hence, maximal effort could have been generated inconsistently. The authors suggested that the EMST device used in the study was designed to strengthen the expiratory muscles, but not specifically in the pattern typically used during cough; therefore, the EMST might have lacked training specificity [22].

\section{Parkinson Disease}

Pitts et al. [4] evaluated the effect of 4 wk of EMST on expiratory muscle strength, voluntary cough, and swallow function in older males with midstage PD. The investigators used Penetration-Aspiration (P-A) Scale [23] to report the degree of penetration/aspiration of thin liquid during videofluoroscopic swallow evaluation. They also measured PEmax and analyzed voluntary cough airflow parameters. Along with a significant increase in PEmax, cough function improved as several cough flow components changed significantly after EMST application. The changes included a major reduction in the mean compression phase duration, expiratory phase rise time, and an increase in cough volume acceleration. The P-A scores also significantly decreased during post-EMST swallow examination, which suggested an improvement in swallow safety [4].

A randomized controlled trial assessing the effect of 4 wk EMST on patients with PD was conducted by Troche et al. [13]. The investigators used physiologic measures of swallow mechanism obtained during barium videofluoroscopic swallow evaluation and assessed the participants' quality of life as related to swallowing function using a dysphagia-specific quality of life (SWALQOL) rating scale. The changes in swallow physiology were assessed based on the hyoid movement and the P-A scores during swallow of a thin liquid. Troche et al. reported a significant post-EMST decrease in P-A scores [13], which suggested an improvement in swallow safety. They also observed substantial increase in hyolaryngeal elevation and excursion during swallow. The maximum hyoid displacement was associated with such events as onset of bolus transit, upper esophageal sphincter (UES) widest opening, UES closure, laryngeal closure, maximum laryngeal closure, and laryngeal opening [13]. In addition, the members of a treatment group demonstrated significant post-EMST improvements on the SWALQOL scale.

\section{Healthy Individuals}

Kim et al. examined the effects of a 4 wk EMST program on PEmax, cough parameters, and cough sensitivity to capsaicin and the relationship between the change in PEmax and cough in healthy but sedentary elderly adults [24]. For the reflexive cough production, the participants inhaled capsaicin or saline solution alternatively. As the cough airflow was analyzed pre- and post-EMST, the investigators observed a posttraining decrease of CPD and PEFR decrease, as well as a postpeak plateau integral amplitude (PPIA) increase. PPIA was defined as the area under cough airflow curve between the initiation and the termination 
of post-peak plateau. All these changes were correlated with a significant increase in PEmax [24]. In the absence of significant changes in either the total number of coughs or expulsive events after EMST, the authors suggested that an increase in reflexive cough strength in the participants was affected primarily by EMST and not by the changes in cough sensitivity to the capsaicin test.

Wheeler et al. examined the timing and amplitude of submental muscle activity during a single EMST trial and during single dry or wet swallows in healthy adults using surface electromyography (sEMG) [25]. They observed longer duration of activation and amplitude of sEMG in the submental muscle group while training on the expiratory device when compared with the swallowing events. The results lead to the conclusion that EMST increases motor unit recruitment of the submental muscle complex [25].

In addition, Wheeler-Hegland et al. assessed hyoid bone displacement during a single trial with an EMST device and during three swallow tasks (normal swallow, effortful swallow, and Mendelsohn maneuver) in healthy adults [26]. The authors expressed an interest in using EMST as a potential non-task-specific dysphagia treatment tool for patients demonstrating inadequate hyolaryngeal excursion and impaired submental muscle function during swallow. The investigated variables included EMG activities of submental muscles, hyoid displacement, and angle of hyoid elevation during the three tasks. Wheeler-Hegland et al. reported significant differences in the trajectory of hyoid motion between tasks with regard to the activation of the submental muscles [26]. All three swallow tasks were associated with greater hyoid displacement than the EMST task, with the maximum angle of hyoid elevation significantly higher for the Mendelsohn maneuver than a normal swallow and highest sEMG values for the effortful swallow. The authors suggested, however, that the EMST task, similarly to an effortful swallow or Mendelsohn maneuver, contributes to increased and prolonged submental muscle activity more than non-exercised swallow tasks [26].

\section{Effect of EMST on Speech Production and Vocal Function}

\section{Lance-Adams Syndrome}

In a single case study, Jones et al. assessed effects of EMST on speech parameters in a 26 yr-old woman with mixed dysarthria as a result of Lance-Adams syndrome (LAS) associated with traumatic brain injury [27]. LAS is a rare condition occurring in patients who have undergone a cardiorespiratory arrest. The syndrome is characterized by action myoclonus that begins within days or weeks after cardiopulmonary resuscitation and persists after a patient recovers consciousness [28].

The outcome measures in the Jones et al. study [27] consisted of maximum phonation time (MPT), intelligibility scores of 14-word sentences, and Communicative Effectiveness Survey (CES) scores. The authors reported immediate post-EMST increase in MPT, speech intelligibility score, and CES score with further improvement of the last measure over the follow-up period. The patient's PEmax was not assessed; therefore, the correlation between PEmax and the other measures could not be analyzed. According to the perceptual assessment, the patient demonstrated laryngeal, lingual, and velopharyngeal weaknesses at baseline. Concerning these impairments, the improvement in speech intelligibility following non-speech-specific training was a particularly interesting outcome. As MPT and speech intelligibility improved after EMST, the authors speculated that the EMST treatment affected those weakened components.

\section{Multiple Sclerosis}

An impact of EMST on PEmax, dysarthria, and voice-related quality of life (VRQOL) in patients with MS and healthy individuals was assessed by Chiara et al. [29], who measured PEmax, sustained vowel prolongation (SVP), words per minute (WPM) in connected speech, and quality-of-life indices related to the presence of the dysarthria and dysphonia after 8 wk of EMST, followed by 4 wk of detraining.

The investigators described the post-EMST increase in PEmax as discussed by Chiara et al. [22]. In addition, they reported the posttraining changes in SVP, which were nonsignificant as a function of the sound pressure level (loud or comfortable). During the detraining period, the sound prolongation at a loud pressure sound level improved significantly in comparison with pretraining assessment. Improvements in speech rates were defined by significantly greater WPM produced at posttraining and detraining than at baseline and a nonsignificant decrease in WPM from posttraining to detraining. Interestingly, there was a reduction in WPM following training and detraining in healthy participants [29]. To evaluate dysarthria-related quality of life in patients with MS and healthy subjects, the investigators used a speech assessment scale, a component of the Amyotrophic Lateral 
Sclerosis Severity Scale (ALSS). This functional assessment tool is typically used by clinicians and is not a patient self-assessment measure. According to the ALSS scores, patients with MS reported significantly less impact of dysarthria immediately after EMST, while patients with moderate MS reported the impact still significantly less after the detraining period. The VRQOL score reflected participants' self-assessment of the impact of dysphonia on their daily activities. Greater improvements in VRQOL scores were reported in participants with MS than in healthy subjects based on the total score and the physical subscale [29].

\section{Professional Voice Users}

To assess the effect of EMST on voice quality, Wingate et al. tested two groups of professional voice users (a non-lesion dysphonia group vs a lesion group), which received both EMST and traditional voice therapy in a different order [30]. The EMST effects were assessed based on three types of ratings: self-rating scales, listener-rating scales for perceptual voice evaluation, and videolaryngostroboscopic ratings. The results included a post-EMST average increase in PEmax, significant reduction of mean Voice Handicap Index scores by 9 points from pre- to midtreatment, reduction in mean scores of the Voice Rating Scale by 83 points between pre- and midtreatment and by 43 points between midand posttreatment, an increase of area of the phonetogram from pre- to posttreatment, and an increase in voice dynamic range from pre- to posttreatment. A significantly higher posttreatment increase in subglottal pressure for higher vocal intensity was reported in the lesion group in comparison with the non-lesion group. No significant differences between groups according to laryngeal diagnosis or a sequence of treatments were observed. Wingate et al. suggested that EMST may serve as an important element of voice therapy to increase subglottal pressure, improve vocal loudness, and reduce voice-related degree of disability [30].

\section{Physiological Responses to EMST}

The safety of therapeutic programs is generally a subject of interest among practitioners using a therapeutic technique with patients. Such an interest has been recently expressed in regard to the EMST, particularly its application in treating patients with cardiovascular diseases. Laciuga et al. tested acute cardiovascular responses to a single EMST session in a group of healthy, young participants [31]. For the purpose of this assessment, the investigators measured blood pressure (BP), heart rate (HR), and oxygen saturation $\left(\mathrm{SpO}_{2}\right)$ before the training session, immediately after the session, and after a $5 \mathrm{~min}$ rest. The secondary measures included a single EMST trial duration. No significant changes in BP, HR, and $\mathrm{SpO}_{2}$ following the EMST trial were observed, which suggested that, although a high expiratory pressure is generated to open the valve within the device, the duration of that pressure generation is relatively short $(1.25 \mathrm{~s})$ and does not require a significant physical and respiratory effort even when the respiratory tasks are repeated [31]. Intrathoracic pressure was not assessed in this study; therefore, the respiratory effort could not be evaluated. Continuous measure of cardiac activity using electrocardiography would have provided more information regarding cardiovascular responses throughout the entire EMST session [31].

\section{DISCUSSION}

The most frequently reported functional outcome of EMST in the selected studies is a significant increase in PEmax, an indicator of expiratory muscle strength $[4,9,11,14-16,22,29-30]$, but other important post-EMST changes have been also documented. Several studies reported a post-EMST decrease of dyspnea perception in healthy participants and patients with COPD during physical activities $[10,12]$ and an increase in a walking distance within a time unit in patients with COPD [6]. Multiple studies demonstrated aerodynamic changes in cough airflow parameters in patients with MS, patients who were elderly, and persons with PD [4,22-23]; improvements in swallow safety in patients with PD $[4,13]$; and post-EMST changes of voice aerodynamic and acoustic parameters [22,27,30]. Adverse events associated with the use of expiratory strength trainers were not reported in the reviewed studies. Due to limited evidence of the safety of EMST in patient groups, caution should be considered when training persons with cardiovascular diseases or demonstrating significant respiratory dysfunction and physical fatigue, because the excessive expiratory load may exacerbate their symptoms.

Noteworthy is the contribution of EMST to improvements in functions such as speech intelligibility and swallow function, which are not directly dependent on expiratory muscle strength or endurance. Assuming that 
activation of the expiratory muscles causes the stimulation of these systems in the motor cortex, EMST may have an improving effect on the activities of adjacent cortical areas. Following this concept, we may conclude that certain processes of neuroplasticity in both the brain and spinal cord can occur as a result of the exercises performed during EMST. More studies comparing EMST with other behavioral strategies or invasive procedures targeting the same impairments should be conducted to identify the most effective treatment for patient care.

\section{CONCLUSIONS}

The EMST program has been successfully applied to increase PEmax, with the potential to improve some aspects of speech, swallow, respiration, and physical performance that require production of adequate expiratory pressure. In the case of voice impairments or dysphagia, the application of EMST as a potential non-task-specific tool may be a future therapeutic approach. Further research should focus on assessing a supportive function for EMST in combination with task-specific exercise programs targeting voice loudness, cough effectiveness, and swallow safety.

\section{ACKNOWLEDGMENTS}

\section{Author Contributions:}

Review concept and design: H. Laciuga.

Literature selection for the review: H. Laciuga, C. M. Sapienza. Approval of selected literature: J. C. Rosenbek, P. W. Davenport. Drafting of manuscript: H. Laciuga.

Revision of manuscript for important intellectual content: C. M. Sapienza, P. W. Davenport, J. C. Rosenbek.

Financial Disclosures: H. Laciuga and J. C. Rosenbek declared that no competing interests exist. C. M. Sapienza has previously received honoraria, including a consultantship with BAE Defense Systems. C. M. Sapienza receives royalties from Plural Publishing. C. M. Sapienza and P. W. Davenport have commercial interest in Aspire Products, LLC, but have received no direct funding from Aspire Products for research. C. M. Sapienza has participated as a co-investigator for the National Institutes of Health, the Department of Veterans Affairs (VA) Office of Research and Development, and industry sponsored trials over the years.

Funding/Support: This material was based on work supported by the Department of Speech, Language, and Hearing Sciences, University of Florida, Gainesville, FL. C. M. Sapienza is a Research Career Scientist with the VA Rehabilitation Research and Development Service. Research on EMST is currently funded by the VA Merit Award program (grant B6576-R).

\section{REFERENCES}

1. Gosselink R, Kovacs L, Ketelaer P, Carton H, Decramer M. Respiratory muscle weakness and respiratory muscle training in severely disabled multiple sclerosis patients. Arch Phys Med Rehabil. 2000;81(6):747-51. [PMID:10857518] http://dx.doi.org/10.1016/S0003-9993(00)90105-9

2. Lahrmann H, Wild M, Zdrahal F, Grisold W. Expiratory muscle weakness and assisted cough in ALS. Amyotroph Lateral Scler Other Motor Neuron Disord. 2003;4(1):49-51. [PMID:12745619] http://dx.doi.org/10.1080/14660820310006733

3. Park JH, Kang SW, Lee SC, Choi WA, Kim DH. How respiratory muscle strength correlates with cough capacity in patients with respiratory muscle weakness. Yonsei Med J. 2010;51(3):392-97. [PMID:20376892] http://dx.doi.org/10.3349/ymj.2010.51.3.392

4. Pitts T, Bolser D, Rosenbek J, Troche M, Okun MS, Sapienza C. Impact of expiratory muscle strength training on voluntary cough and swallow function in Parkinson disease. Chest. 2009;135(5):1301-8. [PMID:19029430] http://dx.doi.org/10.1378/chest.08-1389

5. Polkey MI, Lyall RA, Green M, Nigel Leigh P, Moxham J. Expiratory muscle function in amyotrophic lateral sclerosis. Am J Respir Crit Care Med. 1998;158(3):734-41. [PMID:9730998] http://dx.doi.org/10.1164/ajrccm.158.3.9710072

6. Weiner P, Magadle R, Beckerman M, Weiner M, BerarYanay N. Specific expiratory muscle training in COPD. Chest. 2003;124(2):468-73. [PMID:12907530] http://dx.doi.org/10.1378/chest.124.2.468

7. Larson JL, Kim MJ, Sharp JT, Larson DA. Inspiratory muscle training with a pressure threshold breathing device in patients with chronic obstructive pulmonary disease. Am Rev Respir Dis. 1988;138(3):689-96. [PMID:3202422] http://dx.doi.org/10.1164/ajrcem/138.3.689

8. Smeltzer SC, Lavietes MH, Cook SD. Expiratory training in multiple sclerosis. Arch Phys Med Rehabil. 1996;77(9): 909-12. [PMID:8822685] http://dx.doi.org/10.1016/S0003-9993(96)90281-6

9. Kojima H, Yamada T, Takeda M, Itou Y, Yoshida M, Kimura M. Effectiveness of cough exercise and expiratory muscle training: A meta-analysis. J Phys Ther Sci. 2006; 18(1):5-10. http://dx.doi.org/10.1589/jpts.18.5

10. Mota S, Güell R, Barreiro E, Solanes I, Ramírez-Sarmiento A, Orozco-Levi M, Casan P, Gea J, Sanchis J. Clinical outcomes of expiratory muscle training in severe COPD patients. Respir Med. 2007;101(3):516-24.

[PMID:16942867]

http://dx.doi.org/10.1016/j.rmed.2006.06.024

11. Sapienza CM, Davenport PW, Martin AD. Expiratory muscle training increases pressure support in high school band 
students. J Voice. 2002;16(4):495-501. [PMID:12512637] http://dx.doi.org/10.1016/S0892-1997(02)00125-X

12. Suzuki S, Sato M, Okubo T. Expiratory muscle training and sensation of respiratory effort during exercise in normal subjects. Thorax. 1995;50(4):366-70. [PMID:7785008] http://dx.doi.org/10.1136/thx.50.4.366

13. Troche M, Okun M, Rosenbek J, Musson N, Sapienza C. Swallow outcomes following intervention with expiratory muscle strength training (EMST) in Parkinson's disease: Results of a randomized clinical trial. Dysphagia. 2009;24 (4):455-56.

14. Baker S, Davenport P, Sapienza C. Examination of strength training and detraining effects in expiratory muscles. J Speech Lang Hear Res. 2005;48(6):1325-33.

[PMID: 16478374]

http://dx.doi.org/10.1044/1092-4388(2005/092)

15. Saleem AF, Sapienza CM, Okun MS. Respiratory muscle strength training: Treatment and response duration in a patient with early idiopathic Parkinson's disease. NeuroRehabilitation. 2005;20(4):323-33. [PMID:16403998]

16. Anand S, El-Bashiti N, Sapienza CM. Effect of training frequency on maximum expiratory pressure. Am J Speech Lang Pathol. 2012;21(4):380-86. [PMID:22628108] http://dx.doi.org/10.1044/1058-0360(2012/11-0048)

17. Weiner P, Magadle R, Beckerman M, Weiner M, BerarYanay N. Comparison of specific expiratory, inspiratory, and combined muscle training programs in COPD. Chest. 2003;124(4):1357-64. [PMID:14555566]

http://dx.doi.org/10.1378/chest.124.4.1357

18. Silverman EP, Sapienza CM, Saleem A, Carmichael C, Davenport PW, Hoffman-Ruddy B, Okun MS. Tutorial on maximum inspiratory and expiratory mouth pressures in individuals with idiopathic Parkinson disease (IPD) and the preliminary results of an expiratory muscle strength training program. NeuroRehabilitation. 2006;21(1):71-79. [PMID:16720940]

19. Enright PL, Kronmal RA, Manolio TA, Schenker MB, Hyatt RE; Cardiovascular Health Study Research Group. Respiratory muscle strength in the elderly. Correlates and reference values. Am J Respir Crit Care Med. 1994;149(2 Pt 1):430-38. [PMID:8306041]

http://dx.doi.org/10.1164/ajrccm.149.2.8306041

20. Sasaki M, Kurosawa H, Kohsuki M. Effects of inspiratory and expiratory muscle training in normal subjects. J Jpn Phys Ther Assoc. 2005;8(1):29-37.

http://dx.doi.org/10.1298/jjpta.8.29

21. Griffiths LA, McConnell AK. The influence of inspiratory and expiratory muscle training upon rowing performance. Eur J Appl Physiol. 2007;99(5):457-66. [PMID:17186299] http://dx.doi.org/10.1007/s00421-006-0367-6

22. Chiara T, Martin AD, Davenport PW, Bolser DC. Expiratory muscle strength training in persons with multiple scle- rosis having mild to moderate disability: Effect on maximal expiratory pressure, pulmonary function, and maximal voluntary cough. Arch Phys Med Rehabil. 2006;87(4):468-73. [PMID: 16571384]

http://dx.doi.org/10.1016/j.apmr.2005.12.035

23. Rosenbek JC, Robbins JA, Roecker EB, Coyle JL, Wood JL. A penetration-aspiration scale. Dysphagia. 1996;11(2):93-98.

24. Kim J, Davenport P, Sapienza C. Effect of expiratory muscle strength training on elderly cough function. Arch Gerontol Geriatr. 2009;48(3):361-66. [PMID:18457885] http://dx.doi.org/10.1016/j.archger.2008.03.006

25. Wheeler KM, Chiara T, Sapienza CM. Surface electromyographic activity of the submental muscles during swallow and expiratory pressure threshold training tasks. Dysphagia. 2007;22(2):108-16. [PMID:17294298] http://dx.doi.org/10.1007/s00455-006-9061-4

26. Wheeler-Hegland KM, Rosenbek JC, Sapienza CM. Submental sEMG and hyoid movement during Mendelsohn maneuver, effortful swallow, and expiratory muscle strength training. J Speech Lang Hear Res. 2008;51(5): 1072-87. [PMID: 18728114] http://dx.doi.org/10.1044/1092-4388(2008/07-0016)

27. Jones HN, Donovan NJ, Sapienza CM, Shrivastav R, Fernandez HH, Rosenbek JC. Expiratory muscle strength training in the treatment of mixed dysarthria in a patient with Lance-Adams syndrome. J Med Speech-Lang Pathol. 2006;14(3):207-17.

28. Lee HL, Lee JK. Lance-adams syndrome. Ann Rehabil Med. 2011;35(6):939-43. [PMID:22506225] http://dx.doi.org/10.5535/arm.2011.35.6.939

29. Chiara T, Martin D, Sapienza C. Expiratory muscle strength training: Speech production outcomes in patients with multiple sclerosis. Neurorehabil Neural Repair. 2007;21(3):239-49. [PMID:17351085] http://dx.doi.org/10.1177/1545968306294737

30. Wingate JM, Brown WS, Shrivastav R, Davenport P, Sapienza CM. Treatment outcomes for professional voice users. J Voice. 2007;21(4):433-49. [PMID:16581229]

http://dx.doi.org/10.1016/j.jvoice.2006.01.001

31. Laciuga H, Davenport P, Sapienza C. The acute effects of a single session of expiratory muscle strength training on blood pressure, heart rate, and oxygen saturation in healthy adults. Front Physiol. 2012;3(48):48. [PMID:22419910]

Submitted for publication March 27, 2013. Accepted in revised form December 5, 2013.

This article and any supplementary material should be cited as follows:

Laciuga H, Rosenbek JC, Davenport PW, Sapienza CM. Functional outcomes associated with expiratory muscle 
JRRD, Volume 51, Number 4, 2014

strength training: Narrative review. J Rehabil Res Dev. 2014;51(4):535-46.

http://dx.doi.org/10.1682/JRRD.2013.03.0076

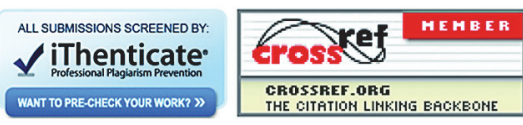

